$\mathbb{3}$
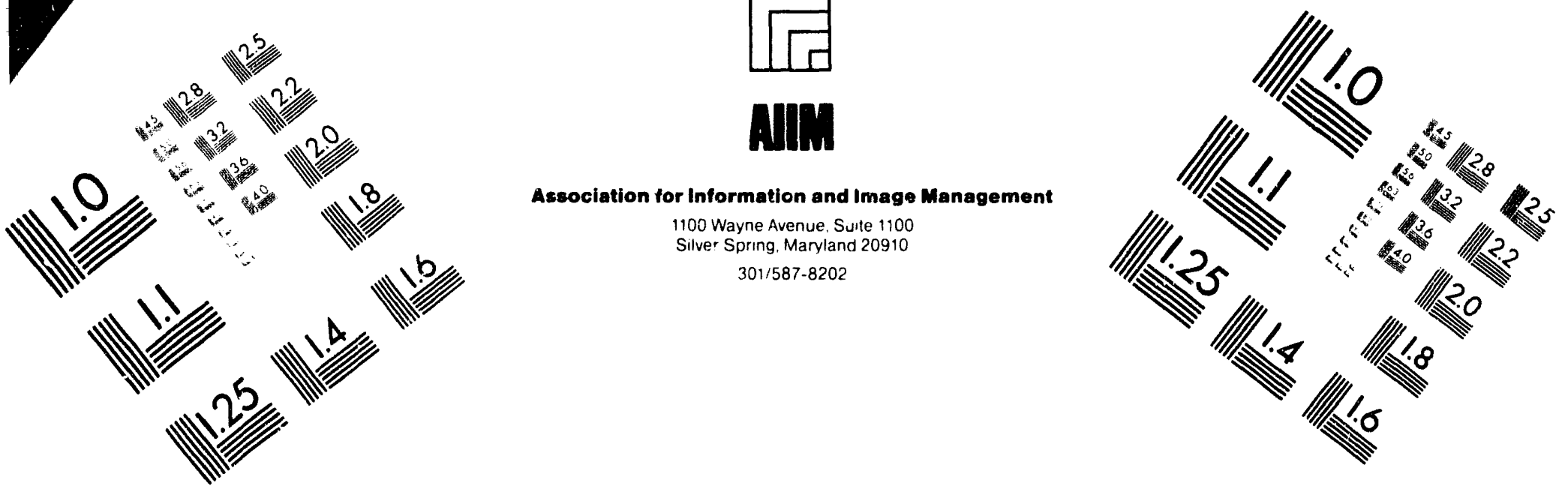

\title{
Centimeter
}

$\begin{array}{llllllllllllllll}1 & 2 & 3 & 4 & 5 & 6 & 7 & 8 & 9 & 10 & 11 & 12 & 13 & 14 & 15 & \mathrm{~mm}\end{array}$ L. Inches
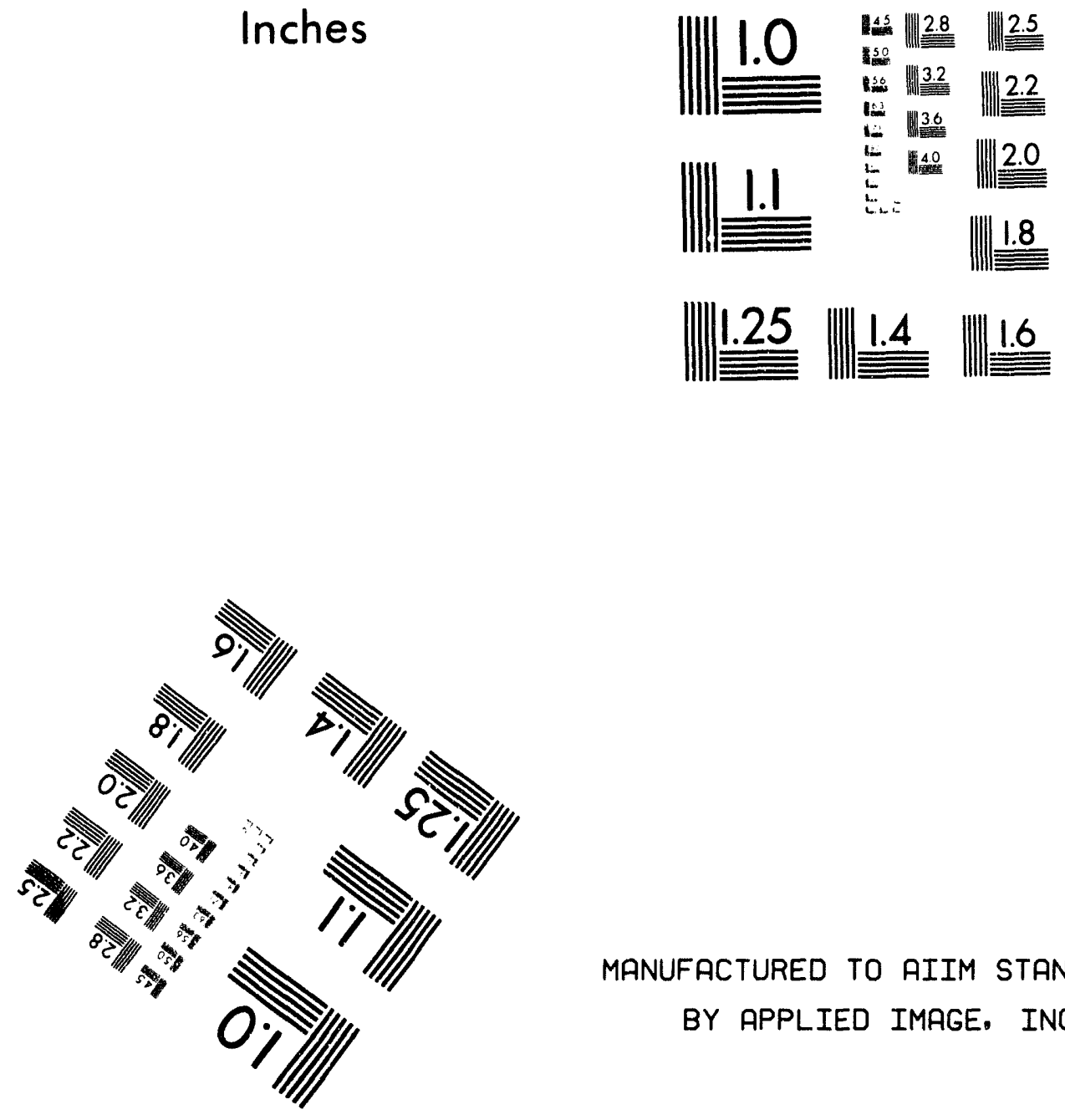

MANUFACTURED TO AIIM STANDARDS BY APPLIED IMAGE, INC.

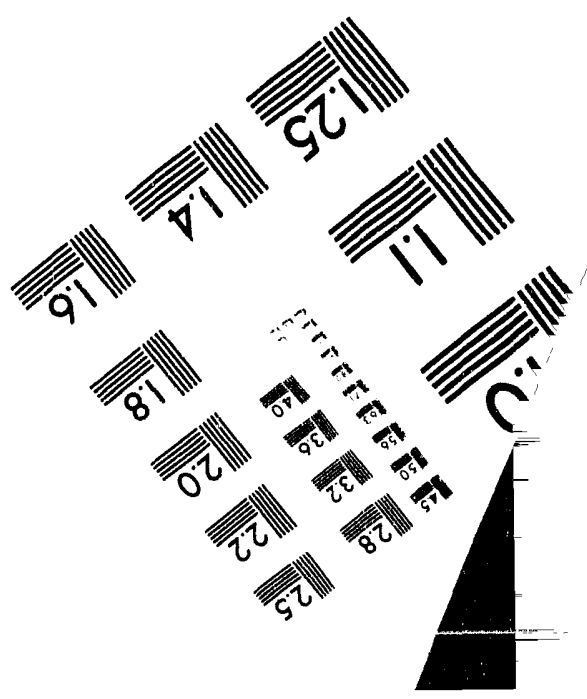



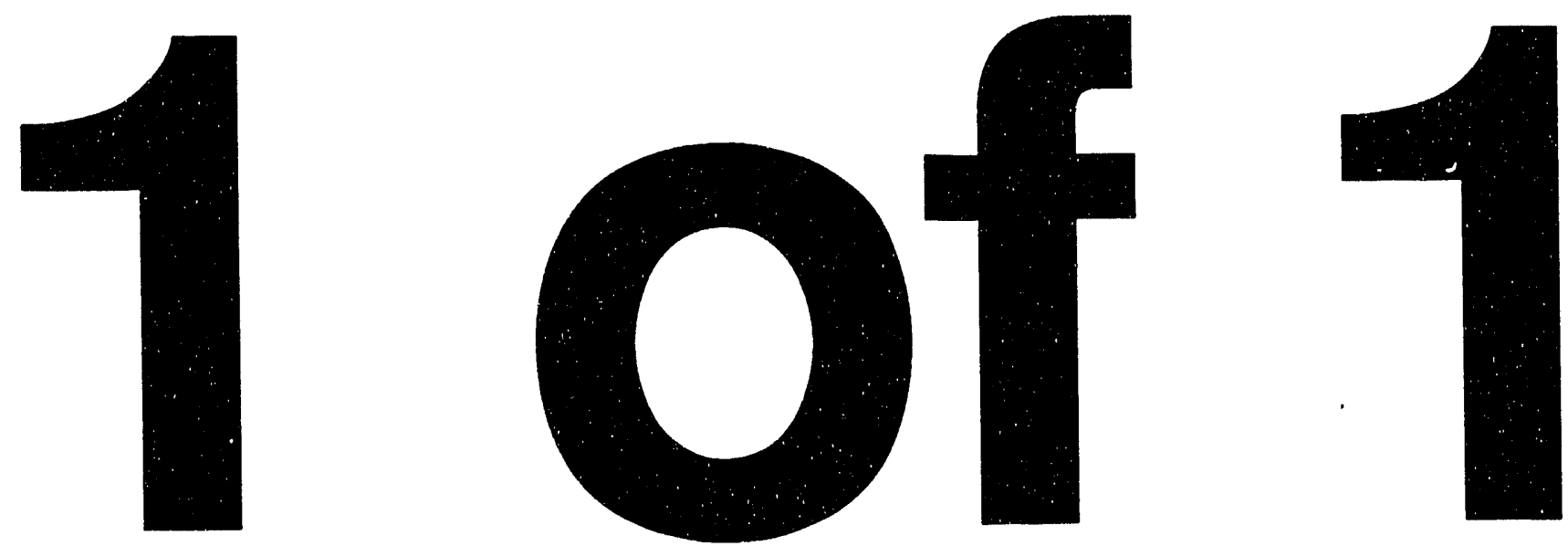
LBL-33256

CBP-226

$\mathrm{UC}-414$

\section{Three-Dimensional Simulation Analysis of the First Sections of a Standing-Wave Free-Electron Laser Two-Beam Accelerator*}

Changbiao Wang and A. Sessler

Lawrence Berkeley Laboratory, University of California, Berkeiey, California 94720

May 1993

* Work supported by the Director, Office of Energy Research, Office of High Energy and Nuclear Physics, Division of High Energy Physics, of the U.S. Department of Energy under Contract No. DE-AC0376SF00098 


\title{
Three-Dimensional Simulation Analysis of the First Sections of a Standing-Wave Free-Electron Laser Two-Beam Accelerator*
}

\author{
Changbiao Wang and Andrew M. Sessler \\ Accelerator and Fusion Research Division, Lawrence Berkeley Laboratory \\ University of California, Berkeley, California 94720
}

\section{Abstract}

A 3-D, time-dependent code is used to simulate an array of standing-wave free-electron lasers (SWFELs) in the two-beam accelerator. It is shown that for an array of SWFELs with 9 cavities and a $100.6-\mathrm{ns}, 0.5-\mathrm{kA}, 7.98-\mathrm{MeV}$ electron beam prebunched at $17.1 \mathrm{GHz}$, an averaged energy output of $14.7 \mathrm{~J} / \mathrm{m}$ can be obtained with a fluctuation of less than $11 \%$.

\section{INTRODUCTION}

In the standing-wave free-electron laser (SWFEL) two-beam accelerator (TBA), ${ }^{1}$ a low energy, high current drive beam alternatively loses its energy for generation of microwave power in standing-wave cavities and has its energy replenished in induction cells. The microwave power is coupled into highgradient $r f$ structures to accelerate an extremely relativistic electron beam of low average current. Hence the drive beam behaves as an energy converter, which converts the energy from the induction cells into of energy. In the TBA configuration, the extraction of microwave power from each free-electron laser (FEL) cavity is small (usually only a few percent) compared to the electron beam power so that the phase-space distribution of the electrons is not severely distorted by the rf fields. Therefore, if the FEL cavities are periodically set in such a way that the energy-phase phase space of the beam before each cavity bas the same distribution, then the fluctuation of the rf ouput powers from these FEL cavities will be small and the output energy per unit length of the array of SWFELs in the TBA becomes relatively stable.

Many studies have been made on the SWFELs in the TBA using the continuum-cavity model $1-3$ and the discrete-cavity model $^{4,5}$ and the impedance-based analysis method. ${ }^{6}$ These treatments are all based on a one-dimensional assumption. Recently, a three-dimensional, time-dependent code, RKFEL, written based on the code RKTW2D, 7 has been used to investigate a multi-cavity FEL. ${ }^{8}$ However, no reacceleration structures were taken into account. In this paper, we use the code RKFEL to study the first sections of a SWFEL/TBA, through which a well-prebunched electron beam is passing. The beam after each cavity is reaccelerated by induction cells so that its average energy remains constant from cavity to cavity. It is shown that for an array of SWFELs with 9 cavities and a 100.6ns, $0.5-\mathrm{kA}, 7.98-\mathrm{MeV}$ electron beam prebunched at $17.1 \mathrm{GHz}$, an averaged energy output of $14.7 \mathrm{~J} / \mathrm{m}$ can be obtained with a fluctuation of less than $11 \%$.

\section{DESCRIPTION OF THE ARRAY OF SWFELS}

So as to be specific, and because of computer cost, for the array of SWFELs we have only used nine identical FEL sections, as shown in figure 1. Each FEL section is $102 \mathrm{~cm}$ ( 6 wiggler periods) long. A beam source provides a $0.5-\mathrm{kA}, 7.98-\mathrm{MeV}$ electron beam with a radius of $3.17 \mathrm{~mm}$. The beam is assumed to have passed through a prebuncher and be well bunched at a

\footnotetext{
* The work was supported by the Director, Office of Energy Research, Office of High Energy and Nuclear Physics, Division of High Energy Physics, of the U. S. Department of Energy under Contract No. DE-AC03-76SF00098.
}

frequency of $17.1 \mathrm{GHz}$ and have a peak if current of $984 \mathrm{~A}$. Then the prebunched beam goes into the FEL sections after it passes a 119-cm drift pipe with a 7-period tapered linear wiggler. In each FEL section (figure 2), there is a cavity with a width of $5 \mathrm{~cm}$, a height of $3 \mathrm{~cm}$, and a length of $86.88 \mathrm{~cm}$, a reacceleration cell 6 $\mathrm{cm}$ long, and a drift pipe $9.12 \mathrm{~cm}$ long. The linear wiggler is uniform and its period is $17 \mathrm{~cm}$. The cavity has a resonance frequency of $17.3 \mathrm{GHz}$ and a wall-dissipation quality factor of 10.000 and an external quality factor of 16 , operating at TE $0.1,96$ mode. The width and beight for all the drift pipes are, respectively, $4 \mathrm{~cm}$ and $0.8 \mathrm{~cm}$.

\section{Prebuncher Tapered Wiggler Drift Region}

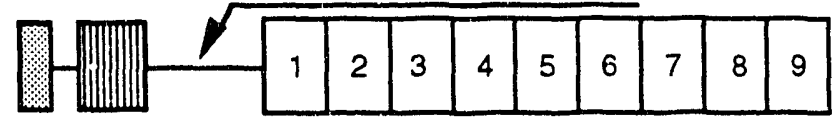

Beam

Source

FEL Sections

Figure 1. Block diagram of an array of the standing-wave free-electron lasers.

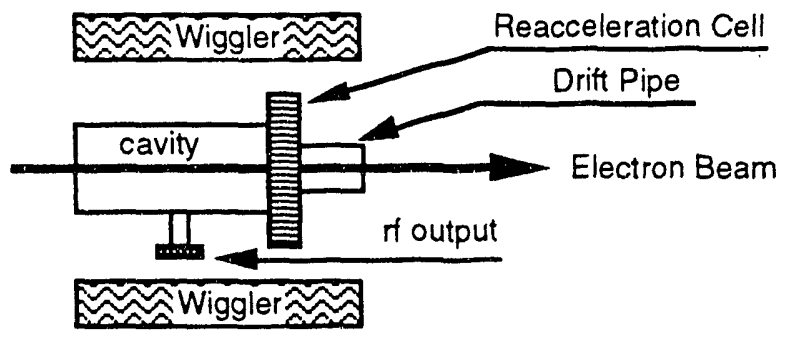

Figure 2. A FEL section.

It should be noted that the length of the FEL section is taken as an integer multiple of the wiggler period to ensure that the beam before each cavity has the same equilibrium distribution in the phase space and thus to reduce variations of output power. In fact, however, the phase-space distributions before the FEL sections can not be made to be exactly the same because of the spread in velocities and the three-dimensional effects of the wiggler magnetic field such as betatron motion.

The vector potential of the linear wiggler magnetic field, provided by parabolically curved magnet pole faces, is given by the following analytic expression ${ }^{9}$

$$
\begin{aligned}
& A_{x}=T(z) \frac{B_{w 0}}{k_{w}} \operatorname{ch}\left(k_{x} x\right) \operatorname{ch}\left(k_{y} y\right) \sin \left(k_{w} z\right), \\
& A_{y}=-T(z) \frac{B_{w 0} k_{x}}{k_{w} k_{y}} \operatorname{sh}\left(k_{x} x\right) \operatorname{sh}\left(k_{y} y\right) \sin \left(k_{w} z\right),
\end{aligned}
$$

where $B_{w 0}$ is the wiggler magnetic field amplitude on the axis, the wiggler wave number is given by $k_{w}=2 \pi / \lambda_{w}$ with $\lambda_{w}$ the wiggler period, $k_{x}^{2}+k_{y}^{2}=k_{w}^{2}$ and the tapering factor is given by $T(z)=z / 7 \lambda_{w}$ when $z<7 \lambda_{w}$, and $T(z)=1$ when $z \geq 7 \lambda_{w}$. When $k_{x}=0$, not only the if field but also the wiggler field is independent of $x$, and the transverse canonical momentum $P_{X}$ is a constant, which is used to check the computational precision. 


\section{SIMULATION RESULTS}

We ran the code RKFEL to simulate the array of the SWFELs with 200 computational particles. The wiggler amplitude $B_{w 0}$ is taken as $0.455 \mathrm{~T}$ with $k_{x}=k_{y}$, corresponding to a detuning frequency of $1.28 \%$. Figure 3 shows the input beam energy and current versus time before the prebuncher. The 100.6-ns pulse has a 4.7-ns rise time, 91.2-ns flat top, and 4.7-ns fall time. We assume that through the prebuncher, a distribution with an initial energy spread $\Delta \gamma /\langle\gamma\rangle=1 \%$ and an initial phase spread $\Delta \omega t$ $=0.2 \pi$ is loaded. where $\gamma$ is the electron relativistic factor and $\omega$ is the drive wave frequency. The 200 particles are randomly distributed within this phase-space rectangle. Figure 4 shows the phase-space distribution for the 50th bucket (about the 60th ns) of the beam right after the prebuncher and before each FEL section. In the tapered wiggler region, the energy spread and the wiggler three-dimensional effect cause increase in the phase spread, and the phase-space rectangle before the tapered wiggler region is changed into a parallelogram before the first FEL section. From the fifth section on, the distributions are quite similar, and the fluctuation of output energy becomes much smaller. The phase spread does not change very much compared with the initial spread. However, simulations indicate that in a state without of fields, the phase spread can become very large with increase in cavity number beacause of the spread in longituciinal velocities. So, the FEL interaction bas the effect of constraining the phase spread at the expense of increasing the energy spread.

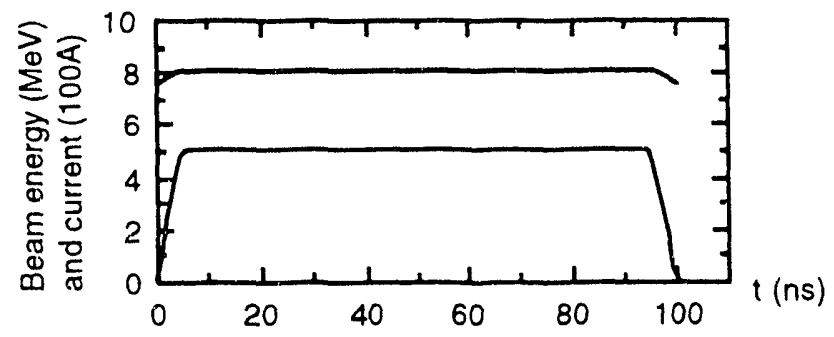

Figure 3. Input beam energy and current versus time.
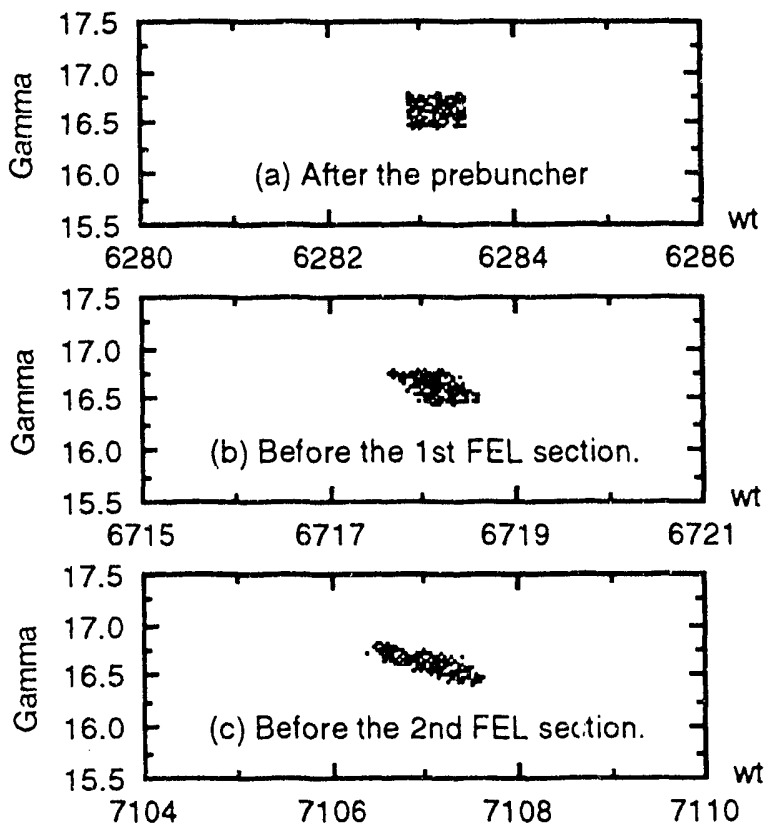
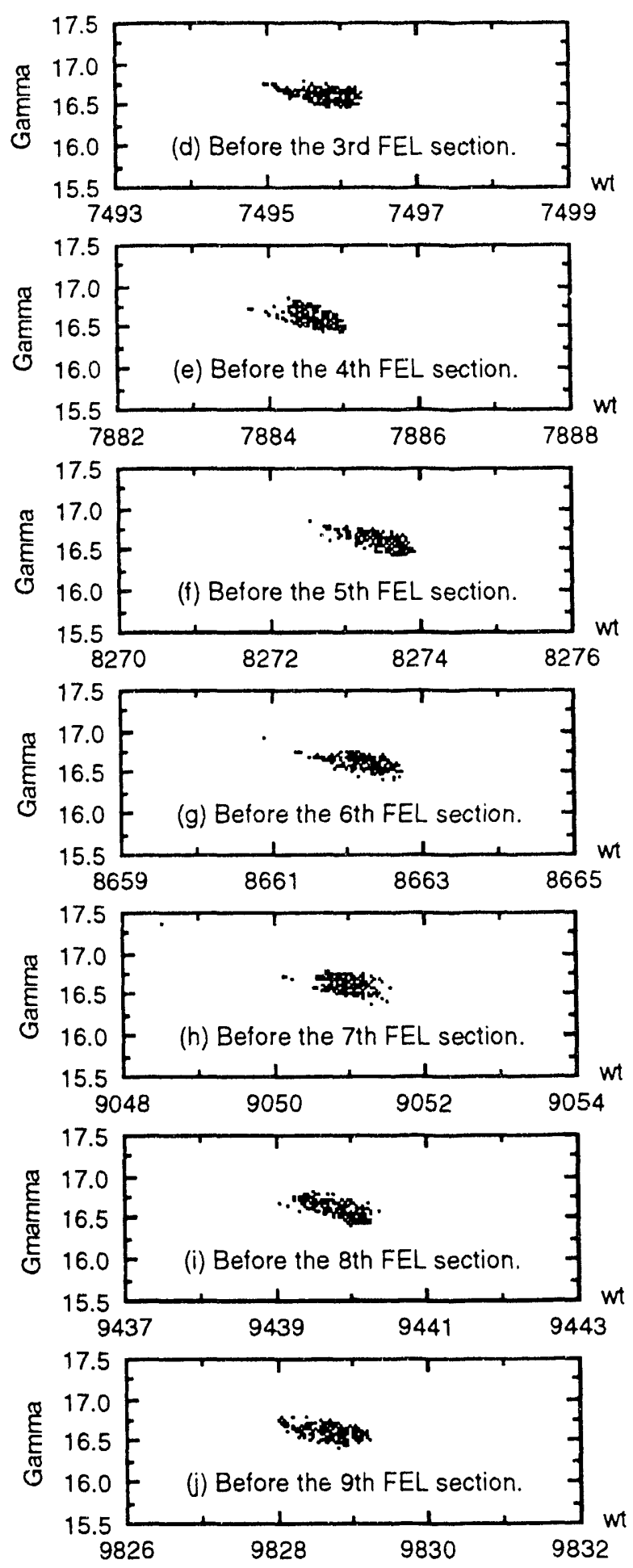

Figure 4. Beam distributions in the $\omega t-\gamma$ space.

Figure 5 shows the dependence of representative cavity output power on time. The output power pulse is a little shortened with increase in the cavity number. However, the power difference between the maximum and minimum is less than $15 \mathrm{MW}$ for the flat part of the pulse. Simulations also indicate that for a too small detuning frequency, the FEL interaction can not lock the phase spread effectively and further, greatly increase the energy spread so that the output power is rapidly decreased with cavities. For a too large 

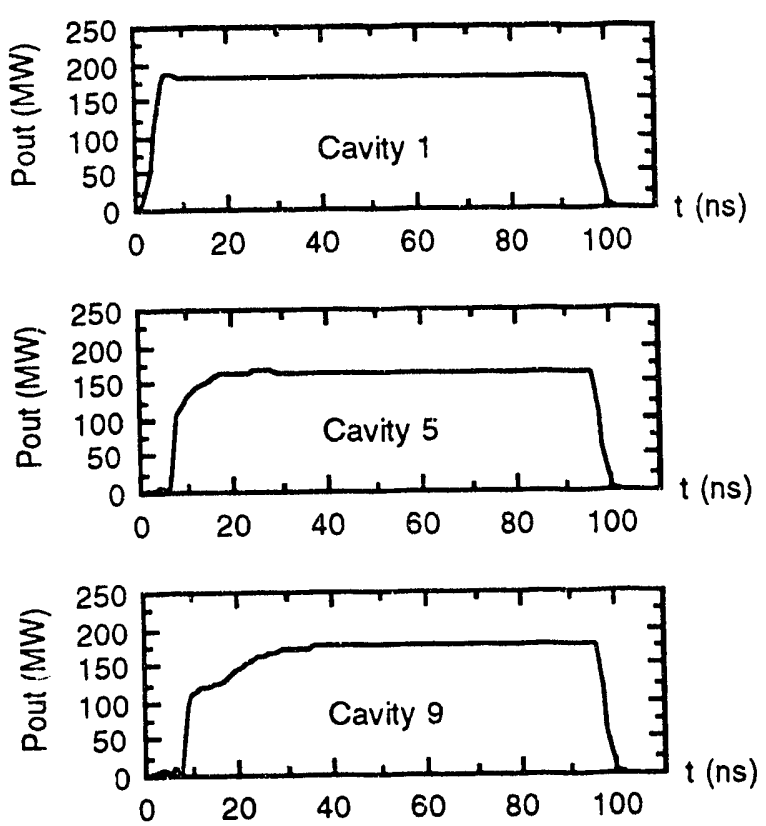

Figure 5. Output power versus time.

detuning frequency, however, the output power for the cavities becomes too small.

Figure 6 shows the electron efficiency and power efficiency versus cavity number for the 50 th bucket. The electron efficiency is defined as $\left.\left.\eta_{e}=\left(<\gamma_{i n}>-<\gamma_{o}\right\rangle\right) /<\gamma_{i n}\right\rangle$, where $<\gamma_{i n}>$ and $\left\langle\gamma_{o}\right\rangle$ are the averaged $\gamma$ before, and after, a cavity. The power efficiency is defined as $\eta_{p}=P_{o u t} / P_{b}$, where $P_{o u t}$ is the output power of a cavity and $P_{b}$ is the input beam power. The electron efficiency is a little greater than the power efficiency because some power is lost on the cavity walls.

Figure 7 shows the output energy and fluctuation versus cavity number (solid circles) during the whole output pulse. The maximum is $16.6 \mathrm{~J}$ and the minimum is $14.4 \mathrm{~J}$. The averaged energy output over the array of FEL sections is $14.7 \mathrm{~J} / \mathrm{m}$. The fluctuation of the output energy is caused by the non-exact periodicity of the electron beam distribution in the phase space. Many factors may influence the periodicity of a beam distribution. The wiggler three-dimensional effect, energy spread, and action of rf fields can all deteriorate the periodicity. To find out how much different the single-particle model and the multi-particle model $^{1}$ are, we ran the code with an initial phase spread of $0.002 \pi$ and a beam radius of $0.0317 \mathrm{~mm}$ ( $1 \%$ of the original ones). As shown in figure 7 (bollow circles), they are in reasonable agreement. The averaged output energy is different only by less than $3.5 \%$ of the original one. Therefore,

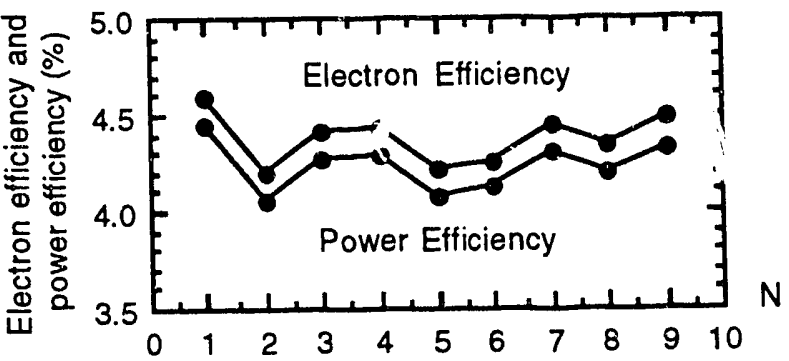

Figure 6. Electron efficiency $\eta_{e}$ and power efficiency $\eta_{p}$ versus cavity number $N$.
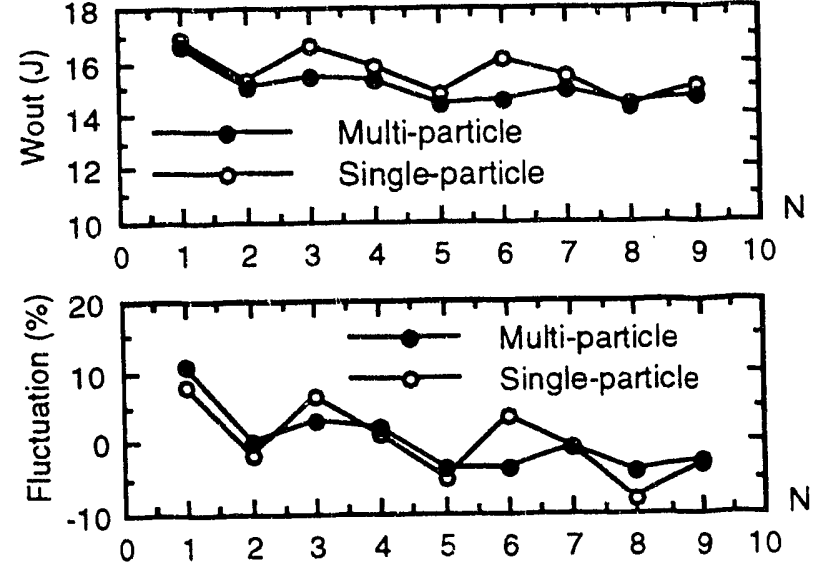

Figure 7. Output energy Wout and fluctuation versus cavity number $\mathrm{N}$.

for a well-bunched beam the single-particle model is a very good approximation

\section{CONCLUSION}

In conclusion, for an array of SWFELs with many cavities in the TBA structure, the phase-space distribution before each cavity becomes quite simalar after the beam passes through only a few cavities, and a stable energy output can be ol,tained.

\section{REFERENCES}

[1] A. M. Sessler, D. H. Whittum, J. S. Wurtele, W. M. Sharp, and M. A. Makowski, "Standing-wave free-electron laser two-beam accelerator," Nucl. Instr. and Meth. A306, 592(1991).

[2] W. M. Sharp, A. M. Sessler, D. H. Whittum, and J. S. Wurtele, "Simulation of a standing-wave free electron laser," Nucl. Instr. and Meih. A304, 487(1991).

[3] W. M. Sharp, G. Rangarajan, A. M. Sessler, and J. S. Wurtele, " Phase stability of a standing-wave free-electron laser," Proc. SPIE Conference 1407, 535(1991).

[4] G. Rangarajan and A. M. Sessler, "Sensitivity studies of a standing-wave free-electron laser," to be publisaed in Proceedings of the Third Workshop on Advanced Accelerator Concepts, June, 1992; Lawrence Berkeley Laboratory Repon LBL-32463, June 1992.

[5] G. Rangarajan, A. M. Sessler, and W. M. Sharp, "Discrete cavity model of a standing.wave free-electron laser," $\mathrm{Nucl}$. Instr. and Meth. A318, 745(1992).

[6] J. S. Wurtele, D. H. Whittum, and A. M. Sessler, "Impecuance-based analysis and study of phase sensitivity in slow-wave two-beam accelerators," to be published in Proceedings of the Thin Workshop on Advanced Accelerator Concepts, June, 1992; Lawrence Berkeley Laboratory Report LBL-3:248, June 1992.

[7] R. D. Ryne and S. Yu, "Relativistic klystron simulations using RKTW2D," Proceedings of the 15th Int'l LINAC Conference, 177(1992).

[8] C. Wang, and A. Sessler, "Three-dimensional simulation analysis of the standing-wave free-electron laser two beam accelerator," to be published in Proceedings of SPIE on Intense Microwave Pulse, January, 1993; Lawrence Berkeley Laboratory Report LBL-32481, January 1993.

[9] E. T. Scharlemann, "Wiggler plane focusing in linear wigglers," J. Appl. Phys. 58, 2154(1985). 

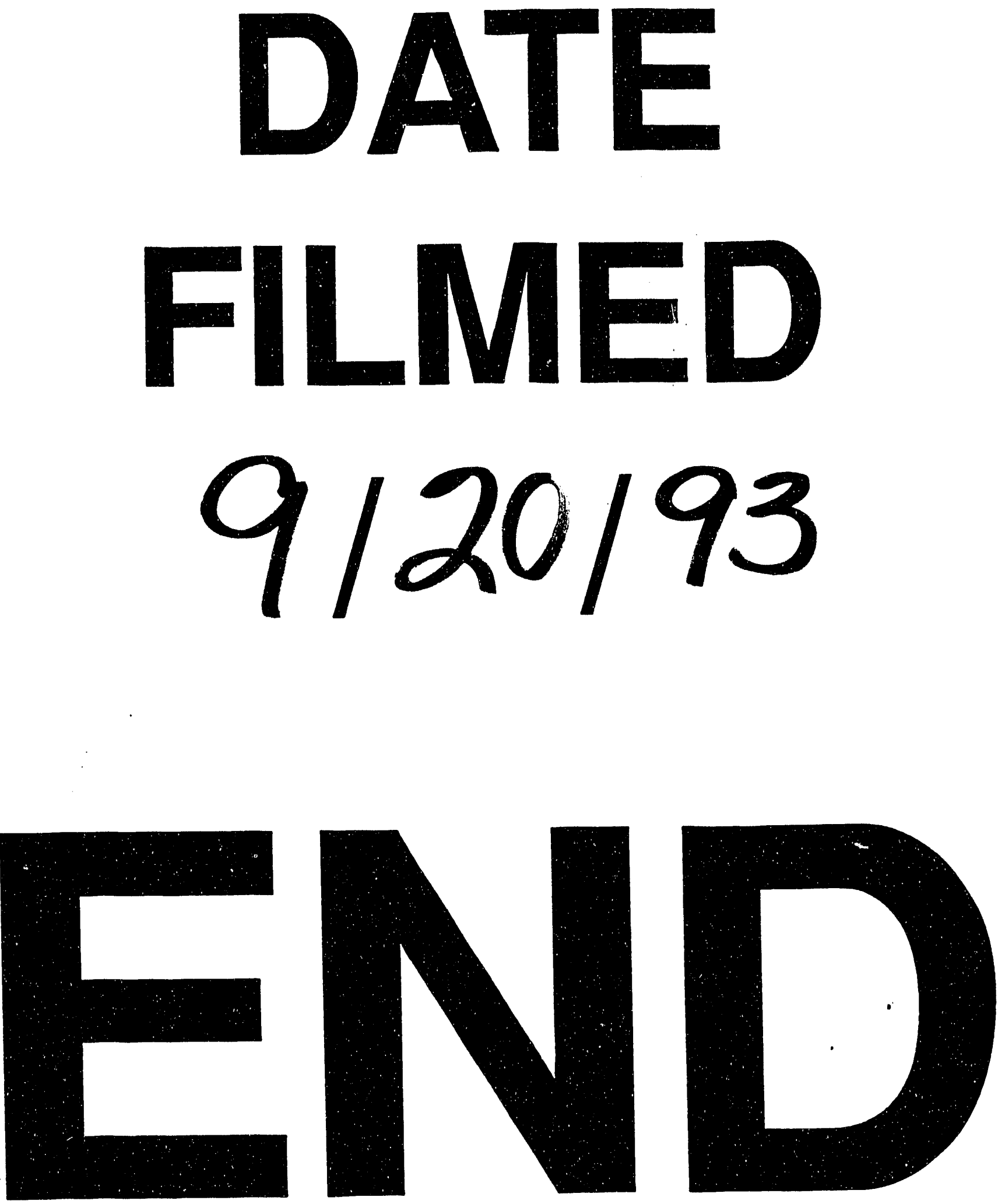
